\title{
Hong Kong Sports Institute: sports medicine and science from theory to practice
}

\author{
Kai-Ming Chan MCh(Orth) FRCS, J. P. S. Wong MBBS, P. Chien FRCS, \\ Ming-Kai Chin PhD, Raymond So MPhil, Josephine Yeung PDPT MPhil, \\ Yvonne Yuan MSc, You-Liang Hong PhD, Mimi Sham BSc and Trisha Leahy MA
} Hong Kong Centre of Sports Medicine and Sports Science, The Chinese University of Hong Kong, Shatin, New Territories, Hong Kong

The Hong Kong Jubilee Sports Centre was established in 1981 as the National Training Centre for élite sportsmen and sportswomen in Hong Kong. There were 11 residential sports: soccer, badminton, table tennis, tennis, fencing, gymnastics, squash, rowing, windsurfing, track and field, and swimming, each with a system of professional coaches and supporting technical staff. A number of non-residential sports were also accommodated within the available facilities or expertise at the centre such as: cycling, judo, shooting, archery, wushu (Chinese martial art), sports for the handicapped and triathlon.

In 1991 there was a major structural change at the Jubilee Sports Centre. As part of the overall evolution of a new system of sports management in Hong Kong the Jubilee Sports Centre was renamed the Hong Kong Sports Institute (HKSI) and now serves as the technical arm of Hong Kong sports for the training and development of élite sportsmen and sportswomen (Figure 1).

There has been a growing awareness in the sporting community that a major impetus for the enhancement of peak performance in athletes stems from an intimate cooperation between coach and athlete on the one hand and technical support of the sports science and sports medicine professionals on the other hand. This formula for success has been well proven in many of the giant sports nations.

\section{A comprehensive care system}

The profile of sports medicine and sports science services included the following: talent identification; surveillance of injuries, diagnosis and treatment; sports rehabilitation; exercise physiology; sports

Address for correspondence: Hong Kong Centre of Sports Medicine and Sports Science, The Chinese University of Hong Kong, 5th Floor, Clinical Sciences Building, Prince of Wales Hospital, Shatin, New Territories, Hong Kong

(C) 1993 Butterworth-Heinemann Ltd 0306-3674/93/020079-06

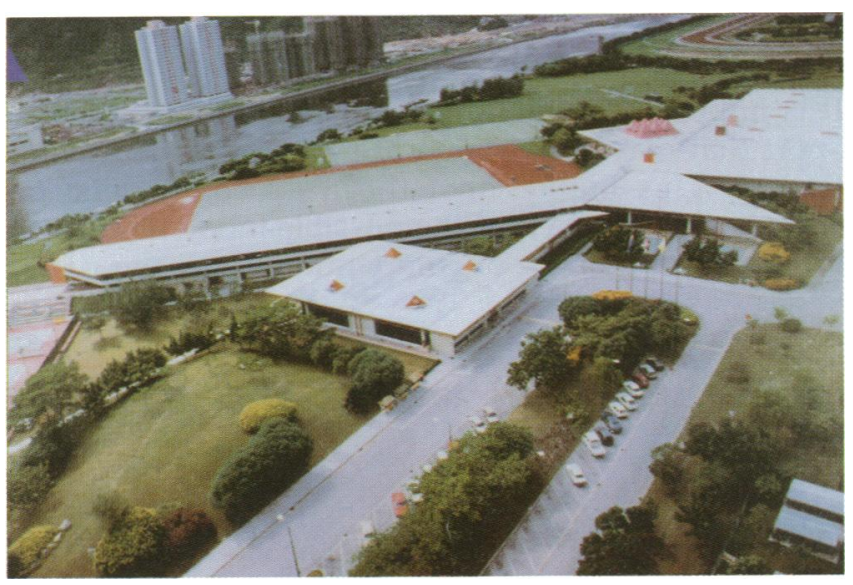

Figure 1. Overview of the Hong Kong Sports Institute

biomechanics; sports nutrition; and sports psychology.

The entire system of comprehensive care obviously demands the cooperation of a team of professionals: sports medicine physician, sports physiotherapist, athletics trainer, strength coach, exercise physiologist, sports nutritionist, sports psychologist, sports biomechanist and sports masseur.

\section{Talent identification}

Specificity in sports training is a well established discipline. Suitable talent for a particular sport should be identified from a pool of young athletes at an early age. This is particularly important in certain sports such as gymnastics, diving and swimming. The ultimate success of this talent identification programme depends on the organization of venue-based sports in schools and sports clubs. At present, we have great difficulty in maintaining a good schools programme in conjunction with an intensive training programme for these sports talents. Due to the lack of a recognized sporting culture and prospect for further career development, there is generally an attitude of 
apathy among the parents. In recent years, through our exchanges with our colleagues in China, we have witnessed tremendous progress in the élite sportsmen training programme arising out of a talent identification strategy. We now recognise that sporting excellence should be nurtured in areas that are more appropriate to the physique and skill of the Southern Chinese such as table tennis, badminton, gymnastics, swimming, squash and wushu.

Once identified, these sporting talents will go through a meticulous assessment programme on their physique profile which includes various anthropometric measurements of body weight, body height, body-fat content, flexibility, cardiorespiratory status, musculoskeletal profile and psychological profile (Figure 2). This screening and assessment will be repeated periodically to identify areas of weakness in order to incorporate appropriate modifications in their overall training programme.

\section{Surveillance of sports injuries, diagnosis and treatment}

It is important to identify risk factors in a particular sport that may lead to overuse injuries or accidental trauma. This implies that the head coach must be adequately informed of the possible mode of injuries arising from training or competition. Over the years,

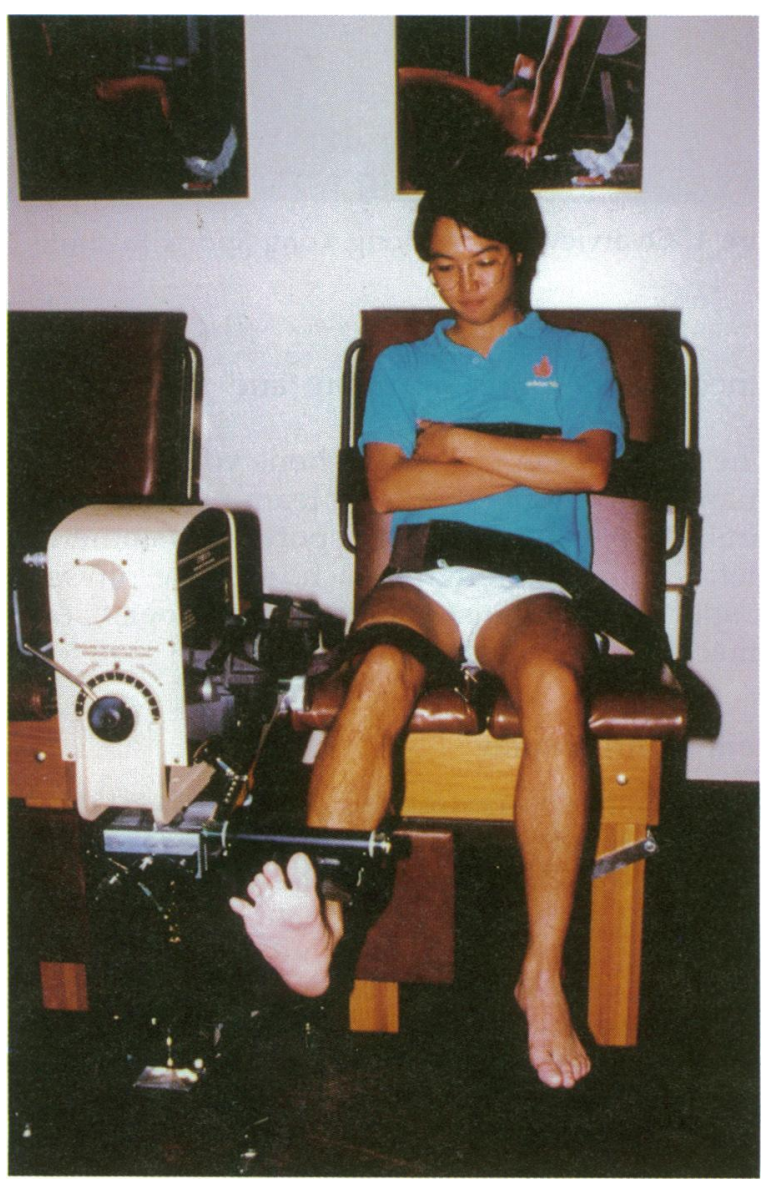

Figure 2. Talent identification - measurement of isokinetic muscular profile through cooperation with the coaches, we have identified specific problems in certain sports and special attention has been paid to designing various preventive measures to minimize the magnitude of the problem. For instance, among the rowers, we have identified an unusually high incidence of low back injury due to weak back and abdominal musculature and also the posture of fixing the spine in the explosive pull of the rowing action. Since then, we have designed a programme of isokinetic assessment of back and abdominal muscles and also instituted video recordings of proper technique and posture of the back to improve the efficiency of the rower. In the meantime, we have also incorporated a general relaxation programme, with regular swimming exercises to improve the strength of upper and lower limb musculature. This has achieved a significant decrease in back problems.

Similarly for some of the racket sports that demand heavy use of the upper arm such as badminton, squash and tennis, we have also identified certain weaknesses of the upper body musculature. This is quite different from the demands required for the throwing action that has been repeatedly described in the literature concerning sports such as baseball. This particular training programme has been well coordinated between the strength coach and the head coach in the respective teams.

The incidence of recurrent ankle sprain among soccer players has been drastically reduced with the enforcement of protective taping of the ankle and a comprehensive ankle rehabilitation programme emphasizing the strengthening of the evertors and proprioceptive re-education training.

The availability of a sports physiotherapy unit within the training centre is a clear advantage in managing many of the soft tissue injuries. The time honoured first aid measure of rest, ice, compression and elevation (RICE) was well observed by the athletes and the coaches. All these injuries were first managed on the spot by the sports physiotherapist to control the immediate pain and swelling, and to prevent further injury with appropriate protection such as taping and use of orthotics. All the injuries were assessed and screened by the consultant orthopaedic surgeon at the weekly sports injury clinic.

An adequate diagnosis of these injuries is a prerequisite for an appropriate therapeutic regime. With the close geographical proximity of the Hong Kong Sports Institute to the Prince of Wales Hospital (the teaching hospital of the Chinese University of Hong Kong), we have an excellent organizational arrangement for sophisticated investigations and supportive treatment programmes. New diagnostic imaging techniques have widened our scope of dignosis:

1. Ultrasonography for delineation of the various soft tissue injuries such as ruptured tendo-Achillis, intramuscular haematoma, shoulder rotator cuff tear and other forms of muscle injuries and chronic tendinitis.

2. The use of radionucleotide scanning has enhanced the diagnosis of stress fracture. 
3. Judicious use of computerized axial tomography (CAT) has proved helpful in the diagnosis of certain problems such as prolapsed intervertebral discs, labrum tears of the shoulder joint and intramuscular haematomas.

4. More recently, the use of magnetic resonance imaging (MRI) has widened our diagnostic horizon in many difficult problems in sports medicine such as prolapsed intervertebral discs with root irritation, anterior cruciate ligament tears, meniscal injuries, and rotator cuff tears of the shoulder.

As more experience is gathered from these new imaging techniques, there will be a reduced need for more invasive investigatory procedures.

For the more severe injuries such as haemarthrosis of the knee or ligamentous instability, the athletes will be referred to the Sports Medicine Unit of the Department of Orthopaedics and Traumatology at the Chinese University of Hong Kong for surgery (Figure 3). In a recent review of 2300 knee arthroscopies performed over an 8-year period, it was revealed that the pattern of arthroscopic surgery included: meniscal injuries (35\%), anterior cruciate ligament tears $(30 \%)$, chondral lesions (chondromalacia patella and degenerative arthritis) $(20 \%)$, synovial lesions and miscellaneous conditions $(15 \%)$. Arthroscopic surgery is also indicated for other joints such as: subacromial decompression for shoulder impingement syndrome, removal of labrum tears and partial rotator cuff tears, removal of loose bodies in the ankle, and assessment of protracted wrist injuries due to triangular fibrocartilage tears, etc. It is clear that the advent of arthroscopic surgery has completely changed our concept of surgical intervention in joint injuries. With this minimally invasive technique it is now possible to deal with well defined problems of the joints and help the athletes return to their preinjury level within the shortest possible time.

\section{Sports rehabilitation}

The concept of rehabilitation should be instituted right from the beginning of the diagnosis of the athletic injury. This is particularly important when

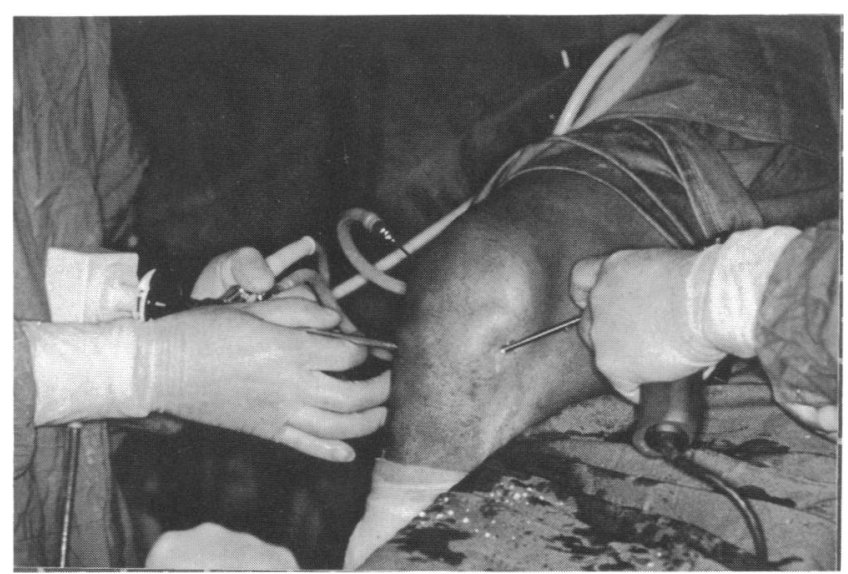

Figure 3. Surveillance of sports injuries, diagnosis and treatment - arthroscopy on the knee élite sportsmen and women are under the tremendous pressure of fulfilling a tight competition timetable. The 'treat and train' principle is emphasized in our programme. While undergoing a course of treatment specific to the injury, an athlete should be maintained at an optimal level of conditioning, particularly with respect to the cardiorespiratory status. For instance, the level of cardiorespiratory fitness of a badminton player with a shoulder impingement syndrome should be maintained through the use of treadmill and bicycle ergometers. At the time, special emphasis is put on the maintenance of the power of the upper body by the use of isokinetic devices and careful control of the angle of movement of the shoulder to avoid the impingement phenomenon. An extensive stretching programme is also utilized to maintain flexibility. Usually at 6 weeks, when there is evidence of early resolution of the irritation, a graduated strength training programme is instituted to restore the upper body strength.

The 'functional progression' programme is widely practised for various types of sports injuries such as a simple ankle sprain, which mainly involves the lateral ligament complex. We start gradual nonweight bearing ankle mobilization as early as $48 \mathrm{~h}$ after the injury when the swelling and pain is under control. Mobility of the ankle joint and various proprioceptive exercises are encouraged. An extensive stretching programme is instituted during this period while the partial tear of the ligament is undergoing gradual healing. Within 4 weeks, we would usually be able to commence a programme of strength training exercises chiefly on the lateral perones and proprioceptive re-education (Figure 4). From 6 weeks onwards, the athletes are instructed to proceed with jogging in a straight line, gradually progressing to circle running and sharp cutting. Full functional training for a specific sport is usually allowed at 10 to 12 weeks. A comprehensive rehabilitation programme, as such, is strictly enforced in order to minimize the risk of recurrence which has previously plagued many of our élite athletes.

\section{Exercise physiology}

The human performance laboratory performs a range of physiological tests, including body-fat assessment, pulmonary function, joint mobility, aerobic and anaerobic power (Figure 5), muscle strength and power, running economy, anaerobic threshold detection and lactate tests. Sports specific tests in rowing, badminton, squash, windsurfing and wheelchair marathon are constructed to assess the specific demands of the sports. The purpose of this work is to provide coaches with more information relating to the intensity of effort of training and competition.

Since its establishment in 1987, physiological tests have been conducted for over 300 HKSI scholarship athletes and Hong Kong teams of 23 sports - archery, badminton, cricket, cycling, disabled track and field, fencing, gymnastics, judo, rowing, sailing, shooting, soccer, squash, swimming, synchronized swimming, 


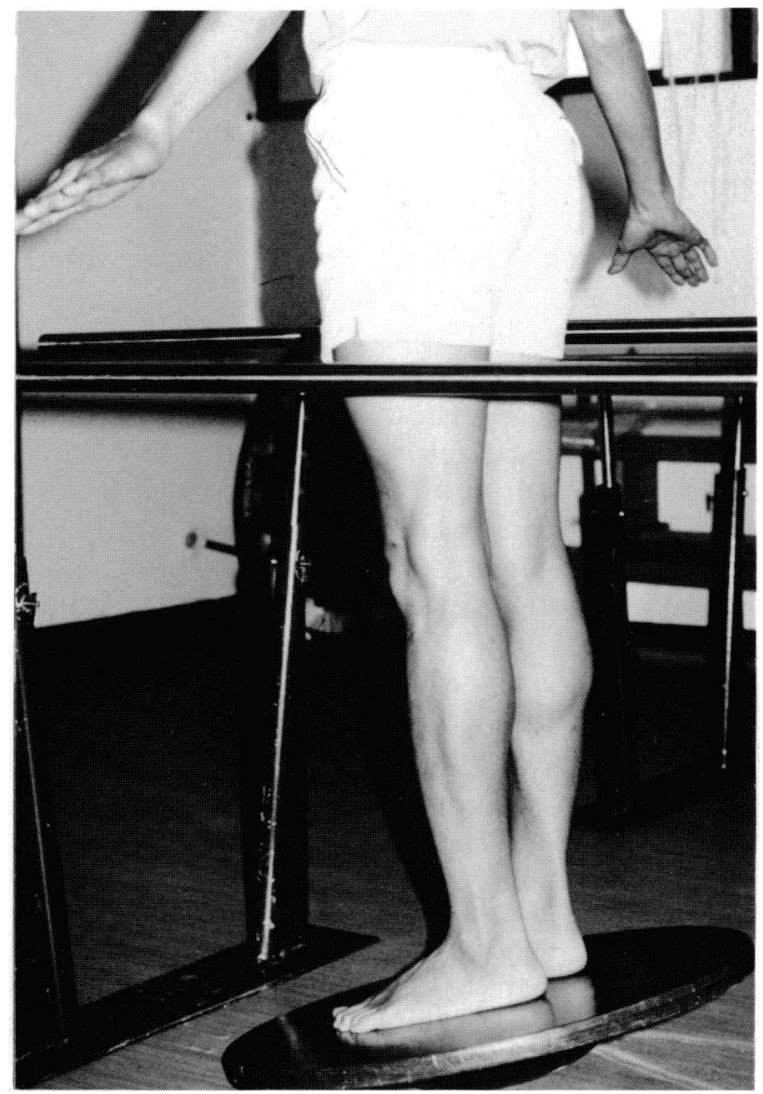

Figure 4. Sports rehabilitation - proprioceptive reeducation for ankle injuries

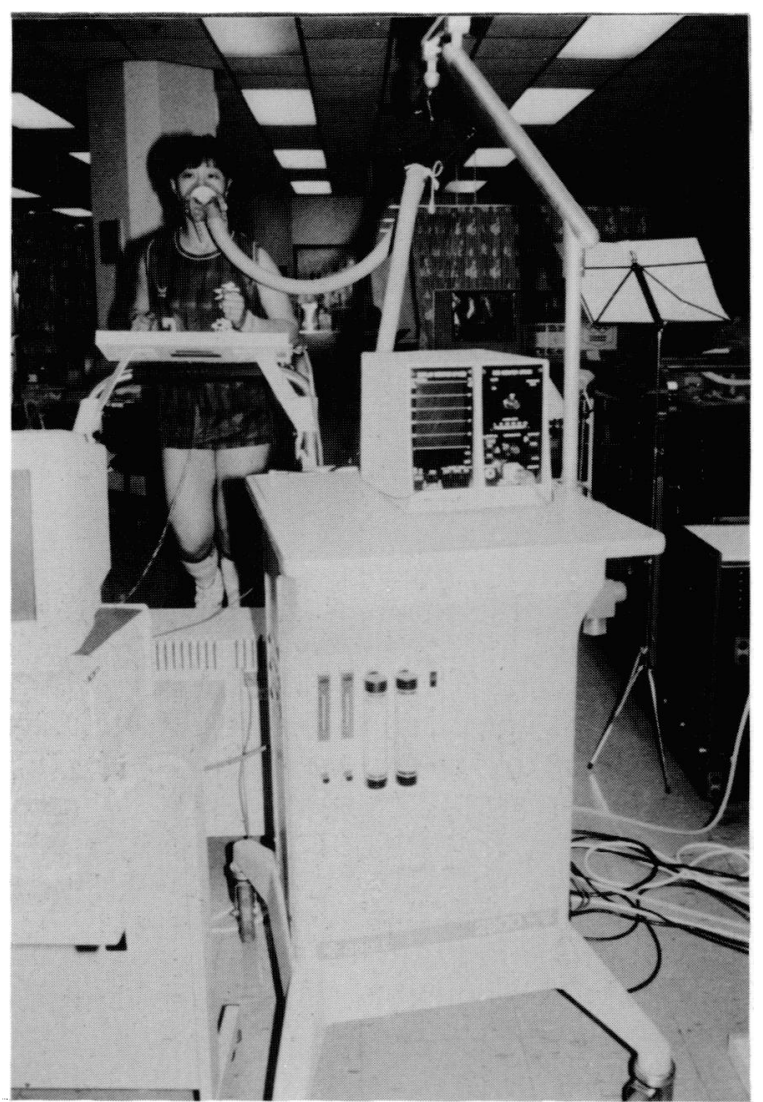

Figure 5. Exercise physiology - assessment of aerobic power table tennis, tennis, tenpin bowling, track and field, triathlon, water polo, wushu and women's basketball. A databank of physiological profiles of various high performance Hong Kong sportsmen and sportswomen has been established on computer so that instantaneous information to coach and athlete can be obtained with high efficiency.

The Executive Top-Fit Program, an extension of the department's range of services to business executives who want to assess and improve their fitness level, was launched in September 1988. Over 60 executives and 30 employees from a large company have undertaken the programme.

\section{Sports biomechanics}

Sports biomechanics has recently been introduced in Hong Kong. Our major goals in sports biomechanics at the HKSI are to educate athletes and coaches in the understanding of specific movement patterns and to help élite athletes improve their peak performance.

In the first stage, kinematic analysis equipment including two video cameras and a peak motion measurement system have been installed at the biomechanical laboratory. A computerized velocity measurement system using photocells is now also under consideration. With the two-dimensional or three-dimensional video filming and the consecutive video type analysis (Figure 6), movement profiles are studied and proposals for performance improvement are discussed with the coaches and athletes in various sports events such as track and field, gymnastics, swimming, tennis, badminton, and squash. To date, we have successfully applied some of these biomechanical principles in training.

For example, the biomechanical study of the sprint run of our $100-\mathrm{m}$ sprinter found insufficient knee extension, significant heel contact of the supporting leg with the ground, as well as too low a position of the swinging leg. The biomechanical analysis also showed that our top long jumper was lacking rhythm and had low velocity in the last four strides of the approach and at takeoff. Three-dimensional analysis

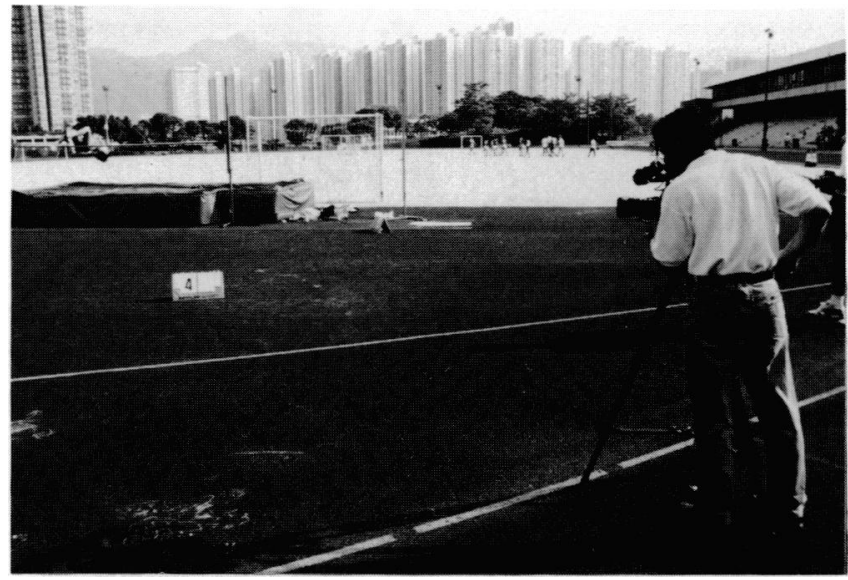

Figure 6. Sports biomechanics - a three-dimensional biomechanical video filming of high jump 
of a badminton smash identified the importance of body rotation and arm flexion for a more powerful smash. With the biomechanical information and specific recommendation to correct or improve sporting techniques, the outcomes are exciting. The sprinter set a new Hong Kong 100-m sprint record, the long jumper improved his rhythm and made accurate board contact and the badminton player increased the shuttle velocity by $22 \%$.

\section{Sports nutrition}

Besides maintaining the well-being of an individual, the importance of optimal nutrition includes maximizing sports performance. At the HKSI, the sports dietitian forms part of the support team. Nutrition service is provided in three major areas: providing consultation to the athletes' food service; individual nutrition counselling (Figure 7); and group teaching.

\section{Athletes' corner}

At HKSI, a secluded dining area is allocated to athletes for the provision of meals. Food items and menus are designed to meet the varied needs of our athletes. With support from the catering department, the provision of a meal service to our athletes in the training ground not only eases athletes' food preparation needs, but also serves as the best medium through which sound nutrition principles are put into practice.

\section{Nutrition counselling}

The nutritional status of an athlete is well reflected by such physiological criteria as: height, weight, body composition, incidence of injury and fitness tests. Athletes are channelled for advice through the coaches or other members of the support team when a nutritional problem is identified. Common nutritional problems encountered by our athletes are: underweight, obesity, poor eating habits and dietary preparation for events. The sports dietitian provides advice and support for achieving dietary behavioural changes.

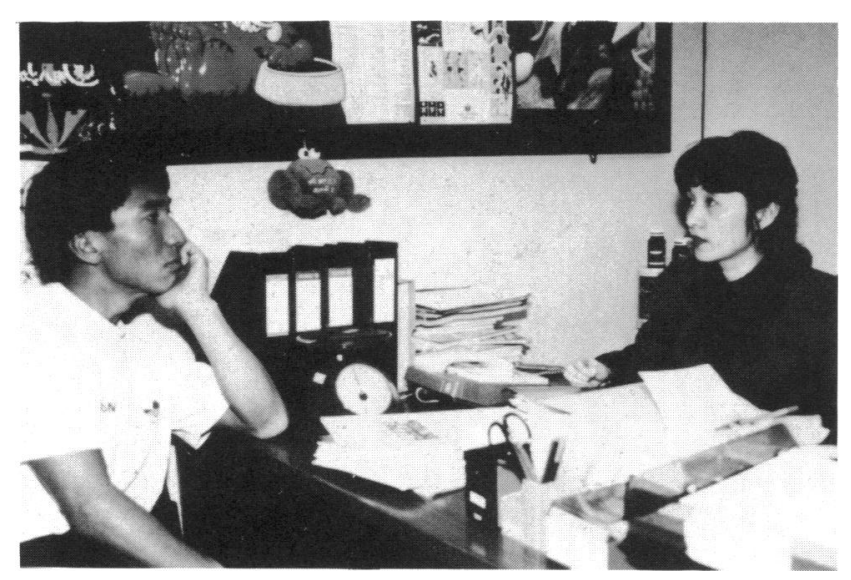

Figure 7. Sports nutrition - individual nutrition counselling

\section{Group learning}

Seminars in sports nutrition are also conducted for individual sports groups and the coaches, with the major objective of improving and updating nutritional knowledge for all those involved in sports.

Recently, a dietary survey was also conducted to assess nutritional attitudes, knowledge and practices of our athletes. Studies similar to this will serve to direct future planning and support in the area of sports nutrition in Hong Kong.

\section{Sports psychology}

Athletic endeavours require enormous amounts of intense involvement. The pressures of high level performance are tremendous. Sports psychology is part of the overall scientific programme of care available to Hong Kong's élite athletes at HKSI. Since the inauguration of the exchange programme with China, a sports psychology expert has been coming to the HKSI for 9 months of each year. The sports psychology unit is in constant communication with the staff of the other sports science units to ensure that a coherent integrated programme is available to the athletes. It is hoped that Hong Kong will soon be able to provide its own sports psychologists. In general terms, the sports psychology unit is concerned with three main aspects:

Education. The sports psychologist conducts both group and individual counselling sessions, and provides lectures to various teams and their coaches, disseminating applied knowledge.

Assessment. The sports psychologist is involved in developing individual databases on the athletes, in response to the need for more understanding of the possible relationship between individual factors, training and performance. These databases are constructed from information obtained through tests, observations and consultations.

Psychological skills training. On the basis of the information obtained through the above, in combination with coach's reports and self reports, HKSI athletes are trained in self-regulation and performance-enhancement skills, including arousal control, relaxation training, biofeedback training, visualization and attention control (Figure 8).

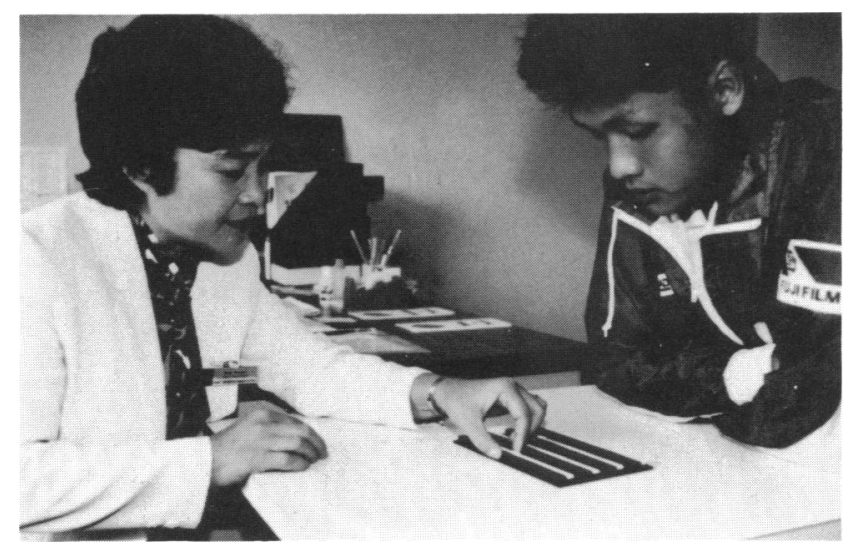

Figure 8. Sports psychology - psychological test for athletes 


\section{Discussion}

The comprehensive system of care for athletics injuries demands careful planning and the implementation of a multidisciplinary team approach. It is also a clear illustration of how sports science and sports medicine can give full support to élite performance. At the HKSI, we have also integrated the elements of research in cooperation with the Hong Kong Centre of Sports Medicine and Sports Science at the Chinese University of Hong Kong and other institutions. Research is the key to progress. From the wealth of data and experience available in the management of sports injuries at the centre, we are now trying to map out an epidemiological profile of sports injuries among our athletes, to refine our diagnostic skill and surgical treatment together with the appropriate rehabilitation programme. The basic scientific research data generated from the various teams in association with other research institutes sheds further light on the experience that we have gathered at field level. This is a pragmatic approach to the application of sports medicine and sports science in sports. We realize that this cooperative venture has maximized our resources and has also given proper recognition of the important role of the individual professional in this field. 